\title{
Consumo de combustível de um trator em função do tipo de pneu, da lastragem e da velocidade de trabalho
}

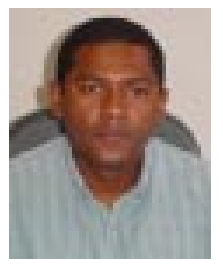

\author{
Afonso Lopes ${ }^{1}$, Kléber P. Lanças ${ }^{2}$, Carlos E. A. Furlani ${ }^{1}$, Alberto K. Nagaoka ${ }^{3}$, \\ Pedro Castro Neto ${ }^{4} \&$ Danilo Cesar C. Grotta ${ }^{1}$
}

\footnotetext{
1 DER/FCAV/UNESP. CEP 14884-900, Jaboticabal, SP. Fone: (16) 3209-2637. E-mails: afonso@fcav.unesp.br (Foto); furlani@fcav.unesp.br, dcgrotta@zipmail.com.br

2 DER/FCA/UNESP. CEP 18603-970, Botucatu, SP. Fone: (14) 6802-7165. E-mail kplancas@fca.unesp.br

3 DER/UDESC. CEP 88520-970, Lages, SC. Fone: (49) 225-2866. E-mail: a2akn@cav.udesc.br

${ }^{4}$ DEA/UFLA. CEP 37200-000, Lavras, MG. Fone: (35) 3829-1362. E-mail: pedrocn@ufla.br
}

Protocolo 150 - 15/10/2002 - Aprovado em 2/7/2003

\begin{abstract}
Resumo: Com o presente trabalho, teve-se como objetivo comparar o consumo de combustível de um trator agrícola operando com pneus (radial, diagonal e de baixa pressão) em duas condições de lastragem (com e sem água nos pneus) e quatro velocidades de deslocamento. $O$ experimento foi realizado na FCAV-UNESP, Jaboticabal, SP, em condição de preparo do solo com escarificador. Utilizou-se um trator $4 \times 2$ TDA, com potência de $89 \mathrm{~kW}(121 \mathrm{CV})$ no motor. Os pneus foram do tipo R1, com as seguintes características: radial (dianteiros: 14.9 R 26; traseiros: 620/75 R 30) diagonal (dianteiros: 14.9-26, traseiros: 23.1-30) e BPAF (dianteiros: 500/60-26.5; traseiros: 700/55-34). O delineamento experimental foi o de blocos casualizados, em esquema fatorial $3 \times 2 \times 4$, com 24 tratamentos e 3 repetições. Os resultados evidenciaram vantagens para o trator quando equipado com pneus radiais. A condição de lastragem com água nos pneus ofereceu menor consumo específico. Observou-se também que o aumento da velocidade de deslocamento reduziu o consumo específico.
\end{abstract}

Palavras-chave: trator agrícola, pneu, consumo de combustível

\section{Fuel consumption of a tractor as a function of the tyre type, ballasting and forward speed}

\begin{abstract}
The objective of this study was to compare the consumption of fuel of the FWD agricultural tractor, equipped with three types of tyres (bias ply, radial and low pressure), with and without tyre water ballasting, operating in four forward speeds. The research was carried out at FCAV/UNESP, SP, Brazil, The soil was tilled using a chisel plow. A tractor with $89 \mathrm{~kW}$ (121 HP) engine power equipped with FWD was used in the experiment, and the tyres used had the following characteristics: radial (front: 14.9R26 and rear: 620/75R30), bias ply (front: 14.9-26 and rear: 23.1-30) and low pressure (front: 500/60-26.5 and rear: 700/55-34). The experiment was conducted in a completely randomized block design $(3 \times 2 \times 4)$, with a total of 24 treatments and three replications. The results evidenced advantages for the tractor equipped with radial tyres. The ballasting condition with water in the tyres showed lower specific consumption. It was also observed that the increase of the forward speed reduced the specific consumption.
\end{abstract}

Key words: agricultural tractor, tyre, consumption of fuel

\section{INTRODUÇÃO}

O sistema de rodado de um trator é o principal elemento responsável pela sustentação, propulsão, direcionamento e desenvolvimento de força na barra de tração, devendo este componente do trator merecer especial atenção. Três níveis de lastragem (100, 90 e $77 \%$ do peso do trator totalmente lastrado) para um trator $4 \times 2$ TDA, operando em pista de concreto, em solo arenoso e em solo argiloso, foram avaliados por Cordeiro (1994) para medir o consumo de combustível. Os resultados mostraram que o consumo de combustível não se modificou quando a carga vertical e as condições do solo se alteraram, mesmo com a retirada de aproximadamente $20 \%$ do seu peso. Lanças et al. (1995) realizaram testes de campo com pneus radiais inflados na pressão correta e com altas pressões, utilizando trator com tração nas quatro rodas e rodado duplo, equipado com pneu 18.4R38. Neste estudo verificou-se que a pressão correta do pneu forneceu melhor desempenho operacional do trator, resultando em menor consumo de combustível. Ensaio comparativo de pneus também foi estudado por Potier (1990) e 
Correa et al. (1997). Esses autores ressaltaram vantagens para o uso de pneu radial.

O consumo de combustível pode ser apresentado como unidade de volume por unidade de tempo $\left(\mathrm{L} \mathrm{h}^{-1}\right)$, caso em que não se considera a influência da variação da temperatura e, tampouco, a quantidade de potência gerada. Outra forma de apresentar o consumo de combustível é por meio de unidade de massa por unidade de tempo $\left(\mathrm{kg} \mathrm{h}^{-1}\right)$; nesta forma, apesar de considerar a influência da temperatura, também não contempla a potência gerada. A forma mais técnica de se expressar o consumo é unidade de massa por unidade de potência $\left(\mathrm{g} \mathrm{kWh}^{-1}\right)$; esta forma é conhecida como consumo específico e, pelo fato de considerar a massa e a potência, pode ser usada para comparar motores, tratores e equipamentos de tamanho e formas diferentes.

Objetivou-se, com o presente trabalho, avaliar o consumo de combustível de um trator agrícola, $4 \times 2$ TDA, variando o tipo de pneu (radial, diagonal e baixa pressão), combinando duas condições de lastragem do trator (com e sem água nos pneus) e quatro velocidades de deslocamento.

\section{MATERIAL E MÉTODOS}

O experimento foi conduzido na FCAV-UNESP, Jaboticabal, SP. A localização geográfica está definida pelas coordenadas 21 ${ }^{\circ} 15^{\prime} 22^{\prime \prime}$ Latitude Sul e 48 18 ' $58^{\prime \prime}$ Longitude Oeste, sendo a altitude média $570 \mathrm{~m}$ e declividade média do solo igual a $7 \%$, apresentando clima tipo Cwa (subtropical) de acordo com a classificação de Köeppen.

Antes do presente estudo, a área experimental estava ocupada por uma cultura de soja (Glycine max L.), sendo a mesma colhida mecanicamente e a massa vegetal $\left(2172 \mathrm{~kg} \mathrm{ha}^{-1}\right)$ distribuída na área pela ação da colhedora. O solo foi classificado como Latossolo Vermelho eutroférrico, A moderado, caulinítico-oxálico e relevo suave ondulado (Andrioli \& Centurion, 1999). No momento dos testes, o teor médio de água no solo foi de $22,8 \%$.

Os pneus de teste foram montados num trator agrícola, $4 \times 2$ TDA, de $89 \mathrm{~kW}(121 \mathrm{CV})$ no motor. O escarificador usado foi do tipo de arrasto conjugado com rolo destorroador e discos de corte, 7 hastes, ponteira sem asa com $7 \mathrm{~cm}$ de largura, trabalhando a $30 \mathrm{~cm}$ de profundidade média. As marchas selecionadas para o ensaio foram denominadas $M_{1}\left(1,84 \mathrm{~km} \mathrm{~h}^{-1}\right)$, $\mathrm{M}_{2}\left(3,18 \mathrm{~km} \mathrm{~h}^{-1}\right), \mathrm{M}_{3}\left(4,57 \mathrm{~km} \mathrm{~h}^{-1}\right) \mathrm{e}_{4}\left(5,04 \mathrm{~km} \mathrm{~h}^{-1}\right)$.

Foram utilizados três conjuntos de pneus de tração do tipo $\mathrm{R} 1$, totalizando 12 unidades, empregados normalmente em tratores agrícolas, os quais são: quatro radiais, quatro diagonais e quatro baixa pressão e alta flutuação (BPAF). Cada conjunto foi formado por dois pneus traseiros e dois pneus dianteiros, cujas principais características técnicas são: radial (dianteiros: 14.9 R 26; traseiros: 620/75 R 30) diagonal (dianteiros: 14.9-26, traseiros: 23.1-30) e BPAF (dianteiros: 500/60-26.5; traseiros: 700/55-34). A instrumentação utilizada nesse trabalho encontrase descrita e ilustrada em Silva (1997) e Lopes (2000).

O trabalho foi conduzido com o trator operando em duas condições de lastragem: lastragem 1: pneus sem água, e lastragem 2: pneus com água, ocupando $75 \%$ do volume. $\mathrm{O}$ trator utilizou, como lastragem fixa, elementos metálicos, sendo seis elos frontais e seis anéis laterais acoplados no centro do rodado traseiro; a massa de cada elemento metálico foi 40 e $50 \mathrm{~kg}$, respectivamente. A massa total do trator, em condição estática, equipado com os pneus de teste, nas duas condições de lastragem, é a seguinte: com pneu radial (sem água $5310 \mathrm{~kg}$, com água $6788 \mathrm{~kg}$ ); diagonal (sem água $5382 \mathrm{~kg}$, com água $6830 \mathrm{~kg}$ ) e baixa pressão (sem água 5624 kg, com água 7060 kg).

\section{Determinação da densidade do combustível}

Utilizou-se o mesmo produto durante o ensaio, sendo o consumo de combustível determinado nas parcelas experimentais em unidade de volume ( $\mathrm{mL}$ ). Juntamente com o volume de combustível, foi monitorada sua temperatura, fornecida em ${ }^{\circ} \mathrm{C}$.

Para se calcular o consumo de combustível em função do tempo ou da potência na barra, foi necessário determinar a densidade do diesel em função da temperatura. Neste procedimento, o intervalo de temperatura foi de 25 a $70{ }^{\circ} \mathrm{C}$; avaliandose a densidade em intervalos de $5{ }^{\circ} \mathrm{C}$ e, por meio de regressão, chegou-se à seguinte equação:

$$
\mathrm{D}=863,22-0,72 \mathrm{~T}
$$

em que:

D - densidade do combustível em função da temperatura, $\mathrm{g} \mathrm{L}^{-1}$

$\mathrm{T}$ - temperatura do combustível, ${ }^{\circ} \mathrm{C}$

$\mathrm{R}^{2}$ - coeficiente de determinação da regressão, 0,9946

\section{Determinação do consumo horário}

Com base no volume consumido, determinou-se o consumo horário em volume e em massa. Para a determinação do consumo horário, expresso em massa, considerou-se a influência da temperatura no momento do teste.

Para o cálculo em volume, utilizou-se a Eq. (2) e para a massa a Eq. (3):

$$
\mathrm{Chv}=(\mathrm{C} \times 3,6) / \mathrm{t}
$$

em que:

Chv - consumo horário, $\mathrm{L} \mathrm{h}^{-1}$

C - volume consumido, $\mathrm{mL}$

$\mathrm{t}$ - tempo de percurso na parcela, $\mathrm{s}$

3,6 - fator de conversão

$$
\operatorname{Chm}=\operatorname{Chv}(863,22-0,72 \mathrm{~T}) / 1000
$$

em que:

Chm - consumo horário com base en massa, $\mathrm{kg} \mathrm{h}^{-1}$

Chv - consumo horário com base em volume, $\mathrm{L} \mathrm{h}^{-1}$

T - temperatura do combustível, ${ }^{\circ} \mathrm{C}$

863,22 e 0,72 - parâmetros da regressão de densidade

1000 - fator de conversão

\section{Determinação do consumo específico}

Este parâmetro expressa o consumo de combustível por unidade de potência na barra de tração. 
Para o cálculo do consumo específico, empregou-se a equação seguinte:

$$
\mathrm{CE}=(\mathrm{D} \times \mathrm{Chv}) / \mathrm{PB}
$$

em que:

CE - consumo específico, $\mathrm{g} \mathrm{kWh}^{-1}$

D - densidade do combustível em função da temperatura, $\mathrm{g} \mathrm{L}^{-1}$

Chv - consumo horário com base em volume, $\mathrm{L} \mathrm{h}^{-1}$

PB - potência na barra de tração, $\mathrm{kW}$

\section{Análise estatística}

O experimento foi conduzido em blocos casualizados, com esquema fatorial $3 \times 4 \times 2$, totalizando 24 tratamentos e 3 repetições. Os tratamentos foram formados pelas combinações de 3 tipos de pneu, 4 marchas do trator e duas condições de lastragem (com e sem água nos pneus). Cada parcela ocupou uma área de $105 \mathrm{~m}^{2}(30$ x 3,5 m) e, entre as parcelas, na direção longitudinal, deixou-se um intervalo de $15 \mathrm{~m}$ para realizar manobras, trânsito de máquinas e estabilizar as determinações. Para comparar-se os tratamentos, avaliaram-se as seguintes variáveis (consumo horário, com base em volume; consumo horário com base em massa e consumo específico). Os dados obtidos foram submetidos à análise de variância, pelo teste $\mathrm{F}$, e a comparação de médias, pelo teste de Tukey, a 5\% de probabilidade. Para o consumo específico procurou-se ajustar um modelo de regressão, validado por meio de análise de variância, pelo teste $\mathrm{F}$, que melhor explica o comportamento dessa variável em função da velocidade de deslocamento.

\section{RESULTADOS E DISCUSSÃO}

\section{Consumo horário em $\mathbf{L} \mathbf{h}^{-1}$}

Pela Tabela 1, percebe-se interação significativa entre os fatores lastro e marcha, o que necessita de desdobramento para explicar os resultados. Analisando-se o fator pneu, verificase que o uso do pneu de baixa pressão resultou em maior consumo quando comparado com os demais; no entanto, os pneus radial e diagonal foram estatisticamente semelhantes.

Analisando-se o fator lastro dentro de cada marcha, constata-se que nas marchas $\mathrm{M}_{1}$ e $\mathrm{M}_{2}$, as duas condições de lastragem foram semelhantes; entretanto, em $\mathrm{M}_{3}$ a condição de lastragem com água resultou em maior consumo, sendo o inverso para a marcha $\mathrm{M}_{4}$ (Tabela 2).

Analisando-se o fator marcha dentro de cada fator lastro, detecta-se que, para a condição sem água, o consumo horário apresentou diferença em todas as marchas ensaiadas, aumentando de $\mathrm{M}_{1}$ para $\mathrm{M}_{4}$; na condição com água, observou-se diferença para as marchas $M_{1}, M_{2}$ e $M_{3}$, sendo $M_{3}$ semelhante a $M_{4}$ (Tabela 2). Os valores encontrados equivalem aos de Furlani (2000), considerando-se que, no referido trabalho, se trata de pneu diagonal, condição de lastragem com água nos pneus e velocidade real de deslocamento semelhante à proporcionada por $\mathrm{M}_{2}$.
Tabela 1. Síntese da análise de variância e do teste de médias ${ }^{\#}$ para as variáveis de consumo horário em volume, consumo horário em massa e consumo específico

\begin{tabular}{|c|c|c|c|}
\hline \multirow[b]{2}{*}{ Fatores } & \multicolumn{3}{|c|}{ Consumo } \\
\hline & $\begin{array}{l}\text { Horário } \\
\left(\mathrm{L} \mathrm{h}^{-1}\right)\end{array}$ & $\begin{array}{l}\text { Horário } \\
\left(\mathrm{kg} \mathrm{h}^{-1}\right)\end{array}$ & $\begin{array}{l}\text { Específico } \\
\left(\mathrm{g} \mathrm{kW} \mathrm{h}^{-1}\right)\end{array}$ \\
\hline \multicolumn{4}{|l|}{ Pneu (P) } \\
\hline Radial & $15,38 \mathrm{a}$ & 12,72 & $476,44 \mathrm{a}$ \\
\hline Diagonal & $15,31 \mathrm{a}$ & 12,65 & $535,85 \mathrm{~b}$ \\
\hline Baixa Pressão & $15,99 \mathrm{~b}$ & 13,65 & $553,67 \mathrm{~b}$ \\
\hline \multicolumn{4}{|l|}{ Lastro (L) } \\
\hline Pneu sem água & 15,48 & 12,86 & $565,18 \mathrm{~b}$ \\
\hline Pneu com água & 15,64 & 12,95 & $478,79 \mathrm{a}$ \\
\hline \multicolumn{4}{|l|}{$\operatorname{Marcha}(\mathrm{M})$} \\
\hline $\mathrm{M}_{1}$ & 10,50 & 8,72 & $725,00 \mathrm{c}$ \\
\hline $\mathrm{M}_{2}$ & 14,24 & 11,82 & $516,74 \mathrm{~b}$ \\
\hline $\mathrm{M}_{3}$ & 18,21 & 15,06 & 435,63 a \\
\hline $\mathrm{M}_{4}$ & 19,29 & 16,01 & 410,57 a \\
\hline \multicolumn{4}{|l|}{ Teste F } \\
\hline $\mathrm{PN}$ & $10,53 * *$ & $15,63 * *$ & $28,14 * *$ \\
\hline $\mathrm{LT}$ & $1,55 \mathrm{~ns}$ & $0,64 \mathrm{~ns}$ & $96,32 * *$ \\
\hline M & $907,30 * *$ & $895,19 * *$ & $262,87 * *$ \\
\hline $\mathrm{P} \times \mathrm{L}$ & $0,70 \mathrm{~ns}$ & $0,65 \mathrm{~ns}$ & $0,84 \mathrm{~ns}$ \\
\hline $\mathrm{P} \times \mathrm{M}$ & $2,26 \mathrm{~ns}$ & $2,36 *$ & $0,68 \mathrm{~ns}$ \\
\hline $\mathrm{L} \times \mathrm{M}$ & $5,57 * *$ & $6,50 * *$ & $1,24 \mathrm{~ns}$ \\
\hline $\mathrm{P} \times \mathrm{L} \times \mathrm{M}$ & $1,88 \mathrm{~ns}$ & $2,16 \mathrm{~ns}$ & $0,89 \mathrm{~ns}$ \\
\hline CV\% & 3,63 & 3,65 & 7,16 \\
\hline \multicolumn{4}{|c|}{$\begin{array}{l}\text { Em cada coluna, para cada fator, médias seguidas de mesma letra minúscula não diferem pelo teste } \\
\text { de Tukey, a } 5 \% \text { de probabilidade, e média desacompanhada de letra implica interação entre os fatores } \\
\text { ns - Não significativo }(p>0,05) \\
* \text { - Significativo }(p<0,05) \\
\text { ** - Significativo }(p<0,01) \\
\text { CV - Coeficiente de variação }\end{array}$} \\
\hline
\end{tabular}

Tabela 2. Desdobramento da interação entre os fatores lastro e marcha para o consumo horário em volume $\left(\mathrm{L} \mathrm{h}^{-1}\right)^{*}$

\begin{tabular}{ccccc}
\hline \multirow{2}{*}{ Lastro } & \multicolumn{4}{c}{ Marchas Ensaiadas } \\
\cline { 2 - 5 } & $\mathrm{M}_{1}$ & $\mathrm{M}_{2}$ & $\mathrm{M}_{3}$ & $\mathrm{M}_{4}$ \\
\hline Sem Água & 10,30 a A & 14,20 a B & 17,79 a C & $19,62 \mathrm{~b} \mathrm{D}$ \\
Com Água & 10,69 a A & 14,29 a B & $18,63 \mathrm{~b} \mathrm{C}$ & 18,96 a C \\
\hline
\end{tabular}

Em cada coluna, médias seguidas de mesma letra minúscula e, em cada linha, médias seguidas de mesma letra maiúscula não diferem pelo teste de Tukey, a $5 \%$ de probabilidade

\section{Consumo horário em $\mathrm{kg} \mathrm{h}^{-1}$}

Pela Tabela 1, percebe-se que, para o consumo com base em massa, ocorreram duas interações significativas, sendo a interação 1: pneu e marcha, e a interação 2: lastro e marcha. A Tabela 3 mostra o desdobramento da interação 1 (fatores pneu e marcha) e a Tabela 4 apresenta o desdobramento da interação 2 (fatores lastro e marcha) para a variável consumo horário em massa $\left(\mathrm{kg} \mathrm{h}^{-1}\right)$.

Analisando-se o fator pneu dentro de cada marcha, notase que na marcha $\mathrm{M}_{1} \mathrm{o}$ consumo horário $\left(\mathrm{kg} \mathrm{h}^{-1}\right)$ foi semelhante

Tabela 3. Desdobramento da interação entre os fatores pneu e marcha para o consumo horário em massa $\left(\mathrm{kg} \mathrm{h}^{-1}\right)^{*}$

\begin{tabular}{lcccc}
\hline \multirow{2}{*}{ Pneu } & \multicolumn{4}{c}{ Marchas Ensaiadas } \\
\cline { 2 - 5 } & $\mathrm{M}_{1}$ & $\mathrm{M}_{2}$ & $\mathrm{M}_{3}$ & $\mathrm{M}_{4}$ \\
\hline Radial & 8,51 a A & 11,44 a B & $14,90 \mathrm{ab} \mathrm{C}$ & $16,01 \mathrm{~b} \mathrm{D}$ \\
Diagonal & 8,77 a A & $11,71 \mathrm{ab}$ B & 14,81 a C & 15,33 a C \\
BPAF & 8,87 a A & $12,32 \mathrm{~b} \mathrm{~B}$ & $15,47 \mathrm{~b} \mathrm{C}$ & $16,70 \mathrm{c} \mathrm{D}$ \\
\hline
\end{tabular}

"Em cada coluna, médias seguidas de mesma letra minúscula e, em cada linha, médias seguidas de mesma letra maiúscula não diferem pelo teste de Tukey, a 5\% de probabilidade 
Tabela 4. Desdobramento da interação entre os fatores lastro e marcha para a variável consumo horário em massa $\left(\mathrm{kg} \mathrm{h}^{-1}\right)^{*}$

\begin{tabular}{ccccc}
\hline \multirow{2}{*}{ Lastro } & \multicolumn{4}{c}{ Marchas Ensaiadas } \\
\cline { 2 - 5 } & $\mathrm{M}_{1}$ & $\mathrm{M}_{2}$ & $\mathrm{M}_{3}$ & $\mathrm{M}_{4}$ \\
\hline Sem Água & 8,58 a A & 11,82 a B & 14,70 a C & $16,33 \mathrm{~b} \mathrm{D}$ \\
Com Água & 8,54 a A & 11,83 a B & 15,41 b C & 15,69 a C \\
\hline
\end{tabular}

Em cada coluna, médias seguidas de mesma letra minúscula e em cada linha médias seguidas de mesma letra maiúscula não diferem pelo teste de Tukey, a $5 \%$ de probabilidade

para todos os pneus estudados. $\mathrm{Na}$ marcha $\mathrm{M}_{2} \mathrm{o}$ pneu radial foi idêntico ao diagonal e apresentou valor menor ao de baixa pressão, mas se notou semelhança entre o diagonal e o de baixa pressão. Na marcha $\mathrm{M}_{3}$ o pneu radial foi semelhante aos demais, e o diagonal teve consumo menor comparado ao de baixa pressão. Na marcha $\mathrm{M}_{4}$ o menor consumo foi observado para o diagonal, seguido do radial e, por último, o de baixa pressão. Analisando-se o fator marcha dentro de cada pneu, observa-se que, para os pneus do tipo radial e de baixa pressão, o consumo horário foi diferente estatisticamente para todas as marchas ensaiadas, para o pneu diagonal; esta variável foi diferente para as marchas $M_{1}, M_{2}$ e $M_{3}$, e $M_{3}$ semelhante a $M_{4}$. $\mathrm{O}$ consumo aumentou de $\mathrm{M}_{1}$ para $\mathrm{M}_{4}$.

Analisando-se o fator lastro dentro de cada marcha, notase que para as marchas $M_{1}$ e $M_{2}$ ocorreu semelhança estatística entre as condições de lastragem; para $M_{3}$ a condição de lastragem sem água resultou em menor consumo e, para $\mathrm{M}_{4}$ a condição sem água apresentou maior consumo. Analisandose o fator marcha dentro de cada lastro detecta-se que para a condição de lastragem sem água ocorreu diferença para todas as marchas. Na condição de lastragem com água observou-se diferença significativa para as marchas $M_{1}, M_{2}$ e $M_{3}$, sendo $M_{3}$ semelhante a $\mathrm{M}_{4}$.

\section{Consumo específico}

Pela Tabela 1, observa-se que o fator pneu influenciou o consumo específico, sendo o menor valor para o pneu radial, enquanto o diagonal foi semelhante ao de baixa pressão. Analisando-se o fator lastro, nota-se menor consumo para a condição de lastragem com água. $\mathrm{O}$ fator marcha teve influência significativa no consumo específico e se notou que esta variável reduziu na medida em que aumentou a velocidade de $M_{1}$ para $M_{2}$ e desta para $M_{3}$, sendo o consumo de $M_{3}$ semelhante $\mathbf{M}_{4}$. Este comportamento está de acordo com Santos Filho (1993), e os resultados equivalem àqueles encontrados por Franz (1988), considerando-se que ambos os autores trabalharam apenas com o pneu diagonal. A Figura 1 mostra o ajuste do modelo de regressão do consumo específico em função da velocidade.

\section{Análise do consumo horário e específico}

Os resultados encontrados neste trabalho oferecem suporte técnico para evitar o consumo horário como base de comparação entre tratamentos, pois o mesmo evidenciou o aumento de velocidade como fator negativo, sendo esta uma inverdade técnica. $\mathrm{O}$ consumo horário com base em volume $\left(\mathrm{L} \mathrm{h}^{-1}\right)$ deve ser usado para se ter uma idéia rápida de campo, lembrando-se que o mesmo não tem amparo científico. $\mathrm{O}$ consumo horário com base em massa considera apenas o efeito da temperatura com a finalidade de corrigir a densidade do líquido.

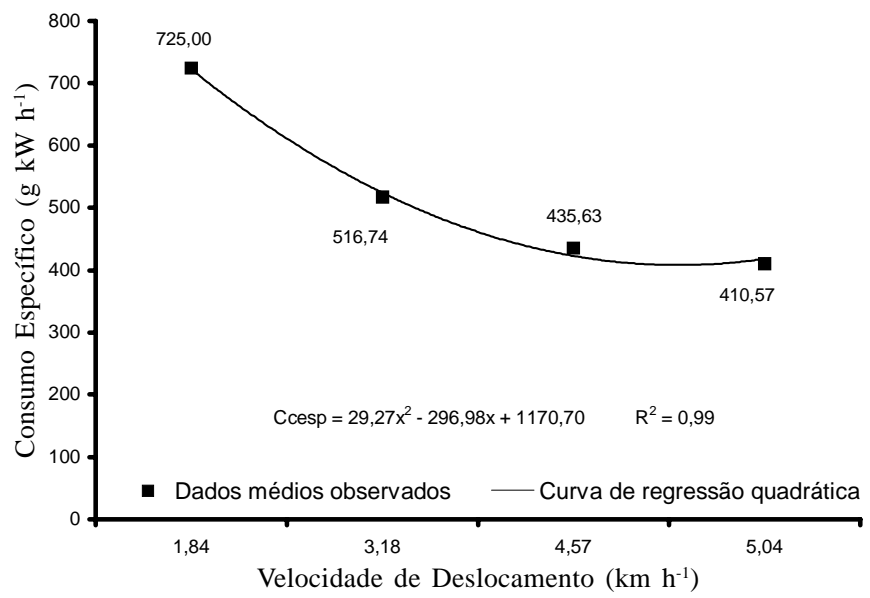

Figura 1. Análise de regressão do consumo específico em função da velocidade

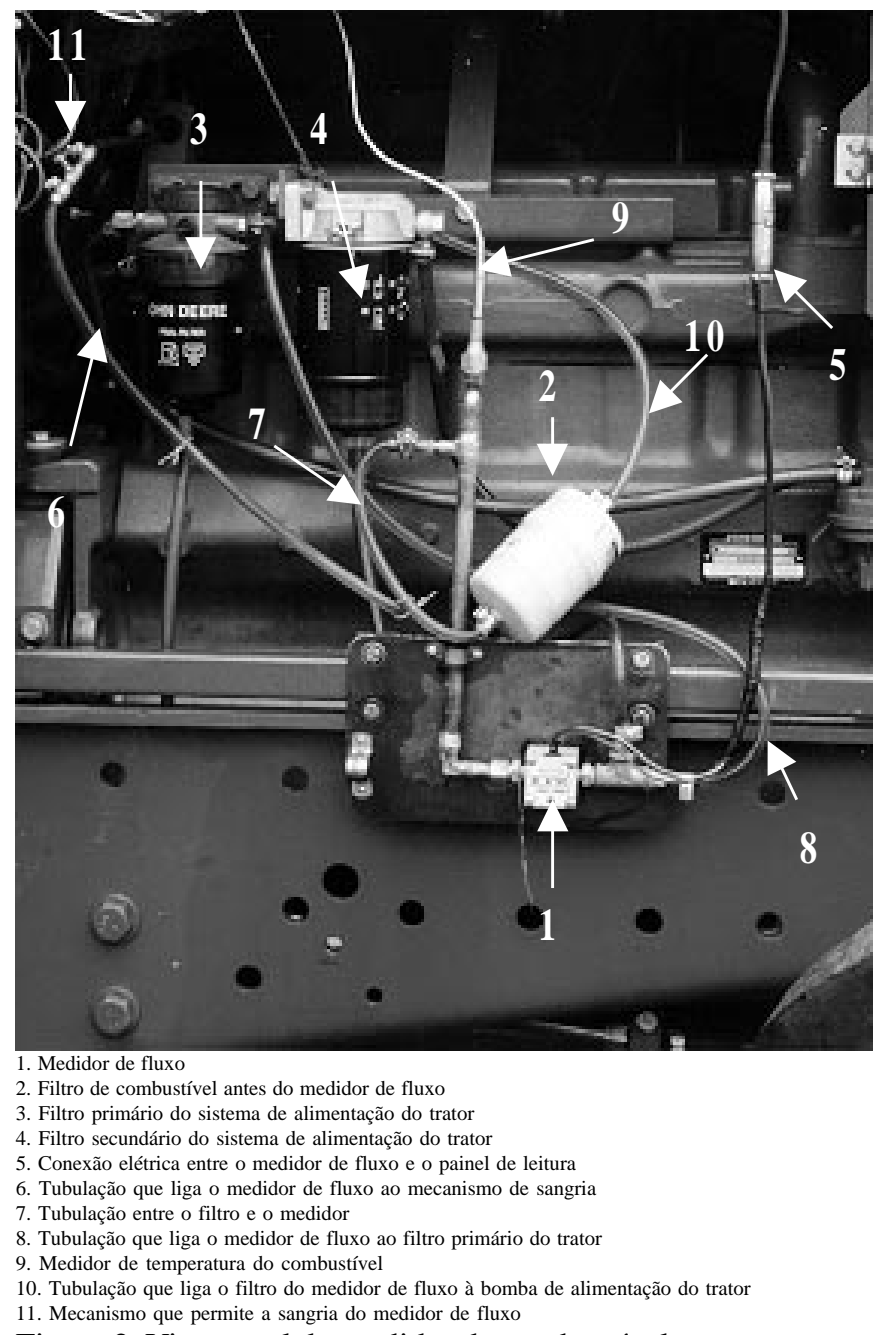

Figura 2. Vista geral do medidor de combustível

\section{CONCLUSÕES}

1. O uso do pneu radial resultou em menos consumo específico.

2. A condição de lastragem com água nos pneu reduziu o consumo de combustível.

3. A velocidade $\mathrm{M} 3\left(4,8 \mathrm{~km} \mathrm{~h}^{-1}\right)$ permitiu reduzir o consumo específico sem comprometer o desempenho ao tratos. 
4. Tratando-se de consumo específico os fatores (pneu, lastragem e velocidade) não interagiram entre si.

\section{LITERATURA CITADA}

Andrioli, I.; Centurion, J.F. Levantamento detalhado dos solos da Faculdade de Ciências Agrárias e Veterinárias de Jaboticabal. In: Congresso Brasileiro de Ciência do Solo, 27, Brasília, 1999. Anais... Brasília, Sociedade Brasileira de Ciência do Solo, 1999. 32p. T025-3 CD-Rom

Cordeiro, M.A.L. Estudo do efeito de lastragem no desempenho de um trator agrícola. Botucatu: Universidade Estadual Paulista. 1994. 112p. Dissertação Mestrado

Corrêa, I.M.; Yanai, K.; Maziero, J.V.G.; Molina Júnior, W.F. Trator agrícola equipado alternadamente com pneu radial de baixa pressão e pneu diagonal: desempenho comparativo. Engenharia Agrícola, Jaboticabal, v.17, n.2, p.64-70, 1997.

Franz, C.A. Avaliação do desempenho de pneumáticos para tratores com diferentes níveis de desgaste. Santa Maria, 1988. 92p. Dissertação Mestrado
Furlani, C.E.A. Efeito do preparo do solo e do manejo da cobertura de inverno na cultura do feijoeiro (Phaseolus vulgaris L.). Botucatu, 2000. 218p. Tese Doutorado

Lanças, K.P.; Santos Filho, A.G.; Upadhyaya, S.K. Implications of using low/correct inflation pressure for radial ply tractor tires. St. Joseph: ASAE, 1995. Paper n.95-056

Lopes, A. Desempenho de um trator agrícola em função do tipo de pneu, da lastragem e da velocidade de deslocamento em um solo argiloso. Botucatu: Universidade Estadual Paulista, 2000.131p. Tese Doutorado

Potier, M. Pneus: la pression baisse. Agri-Decideur, n.26, p.1528, 1990.

Santos Filho, A.G. Desempenho energético e operacional em função da velocidade de deslocamento e teor de água no solo. Botucatu, 1993. 141p. Dissertação Mestrado

Silva, S.L. Projeto e construção de sistema de aquisição de dados para avaliação do desempenho energético de máquinas e implementos agrícolas. Botucatu: Universidade Estadual Paulista, 1997. 148p. Dissertação Mestrado 